\title{
PREFERÊNCIAS DE ATIVIDADES DE LAZER DE UM GRUPO DE IDOSAS DO EXTREMO SUL DO BRASIL
}

Recebido em: 22/11/2012

Aceito em: 20/05/2013

\section{Luiza Isnardi Cardoso Ricardo ${ }^{1}$ \\ José Antônio Bicca Ribeiro ${ }^{1}$ \\ Rodrigo Wiltgen Ferreira ${ }^{2}$ \\ Adriana Schüler Cavalli ${ }^{3}$}

Escola Superior de Educação Física

Universidade Federal de Pelotas

Pelotas - RS - Brasil

RESUMO: A população idosa no Brasil está aumentando de forma acentuada. Sendo assim, investigações acerca da complexidade do processo de envelhecimento tornam-se imprescindíveis. O lazer é benéfico à manutenção da saúde, no desenvolvimento biopsicossocial dos idosos, principalmente por estabelecer relações com o meio onde vivem. O presente estudo tem como objetivo verificar as preferências de atividades de lazer de idosas participantes do projeto NATI-ESEF-UFPel. Este estudo descritivo de corte transversal foi desenvolvido por meio de um questionário semi-estruturado, aplicado em 191 idosas participantes do projeto. Os resultados apontam que as idosas têm preferência por atividades de lazer que possam ser realizadas em seu domicílio, predominando as atividades artísticas e manuais.

PALAVRAS CHAVE: Atividades de Lazer. Idoso. Socialização.

\section{LEISURE ACTIVITIES' PREFERENCES OF AN ELDERLY WOMEN GROUP FROM SOUTHERN BRAZIL}

ABSTRACT: The elderly population in Brazil is growing intensely. Therefore, surveys about the complexity of the aging process has become essential. Leisure is essential on the maintenance of health, as well as older people's self-worth, mainly by establishing relationships in the environment. The present study aims to determine the preferences of leisure time activities of the elderly, participants in the NATI-ESEF-UFPel project. This cross-sectional survey was developed through a semi-structured questionnaire, administered to 191 elderly participants of the project. The results indicate that the

\footnotetext{
${ }^{1}$ Mestrandos do Programa de Pós-Graduação em Educação Física da ESEF/UFPel.

${ }^{2}$ Acadêmico de Educação Física da ESEF/UFPel. Bolsista do Programa de Educação Tutorial.

3 Docente dos cursos de Graduação e Pós-Graduação em Educação Física da Escola Superior de Educação Física (ESEF/UFPel).
} 
majority of the elderly prefer leisure tome activities that can be performed at home, predominantly artistic and manuals activities.

KEYWORDS: Leisure Activities. Aged. Socialization.

\section{INTRODUÇÃO}

A população idosa do Brasil está crescendo de forma acelerada e intensa. Dados do Censo de 2010 do Instituto Brasileiro de Geografia e Estatística (IBGE, 2010) apontam que a população de idosos no Brasil representa $11 \%$ da pirâmide etária total, com o equivalente a 20.590.599 de pessoas em números absolutos. Em contrapartida, no Censo anterior realizado no ano de 2000, o número absoluto de idosos era igual a 14.536.029, havendo um aumento de 29,4\% em um período de dez anos.

Em resposta a esta transição demográfica, a investigação acerca da complexidade do processo de envelhecimento tornou-se imprescindível, sendo um dos principais temas de pesquisa de diversas áreas do conhecimento. Estas pesquisas procuram compreender aspectos e dimensões biológicas, psicológicas, sociais e culturais que venham a contribuir positivamente para o processo de desenvolvimento humano ao longo da vida do idoso (PRADO; SAYD, 2004; GÁSPARI; SCHWARTZ, 2005).

As perdas orgânicas decorrentes do envelhecimento tais como, limitações físicas e cognitivas, podem contribuir para uma diminuição da capacidade funcional do idoso, afetando-os na realização dos afazeres diários e autocuidados. Em contrapartida, existem evidências de que idosos que se mantêm ativos na sociedade, envolvidos em diversas atividades, têm maiores chances de conservar sua auto-estima e autonomia (SANTOS; FORONI; CHAVES, 2009). 
Uma das melhores, e mais procuradas maneiras para manter a independência do idoso é proporcionando atividades de lazer e de socialização para o mesmo. O lazer é essencial à manutenção da saúde, bem como à autovalorização das pessoas idosas, principalmente por estabelecer relações com o meio ambiente, condição esta que favorece o inter-relacionamento das pessoas, contribuindo, de certa forma, para se viver melhor com os outros e para o bem-estar de cada um (MARCELLINO, 2006; DAVIM; et al., 2003).

Especificamente no Brasil, diversas ferramentas são utilizadas para garantir o direito do idoso ao lazer. A primeira delas data de quatro de janeiro de 1994, quando foi aprovada a Lei $\mathrm{n}^{\mathrm{o}} 8.842$, que dispõe sobre a Política Nacional do Idoso, com o objetivo de assegurar os direitos sociais do idoso, criando condições para promover sua autonomia, integração e participação efetiva na sociedade. Além disso, visava incentivar os idosos a desenvolver atividades culturais e incentivar a criação de programas de lazer, esporte e atividades físicas que proporcionem a melhoria da qualidade de vida do idoso e estimulem sua participação na comunidade (BRASIL, 1994).

A partir desta lei, que foi reforçada pelas leis $n^{\circ} 10.741$ de $1^{\circ}$ de outubro de 2003 (BRASIL, 2004), juntamente com a lei nº 2.528 de 19 de outubro de 2006 (BRASIL, 2006), diversas instituições universitárias do país vem desenvolvendo trabalhos visando à valorização do idoso como agente integrante da sociedade, através do fortalecimento da participação social do idoso, contribuindo para a formação de um cidadão consciente de seus direitos e deveres (FENALTI; SCHWARTZ, 2003).

Neste sentido, pensando em oferecer oportunidade para os idosos participarem de atividades físicas e de lazer, a Escola Superior de Educação Física (ESEF) da Universidade Federal de Pelotas (UFPel) criou, em 1993, o Núcleo de Atividades para a 
Terceira Idade (NATI). Atualmente, este projeto social oferece ao público idoso a prática de atividades físicas orientadas em diversas modalidades, assim como atividades culturais, atendendo aproximadamente 500 indivíduos da cidade de Pelotas/RS (AFONSO; et al., 2010), sendo um importante espaço de atuação para os acadêmicos do curso de Educação Física, contribuindo para a sua formação acadêmica e profissional (PINTO, 2001).

O presente estudo tem como objetivo verificar as preferências de atividades de lazer das idosas participantes do projeto NATI-ESEF-UFPel.

\section{METODOLOGIA}

Este estudo configura-se como descritivo de cunho transversal, em que utilizouse um questionário semi-estruturado elaborado pelo Grupo de Pesquisa e Estudos Sociológicos em Educação Física e Esportes (GPES-UFPel), possuindo questões divididas em três eixos principais: a) dados de identificação do participante e histórico de doenças/hábitos de vida; b) atividade física e de lazer na vida do participante; c) nível socioeconômico do participante segundo a Associação Brasileira de Empresas de Pesquisa - ABEP.

O questionário foi aplicado de forma individual, pelos acadêmicos participantes do projeto, sob a supervisão das coordenadoras, na Escola Superior de Educação Física. A questão referente às atividades de lazer foi composta por uma lista de atividades em que as idosas poderiam assinalar todas as opções de sua preferência. Atividades não listadas puderam ser descritas na opção outras com a devida exemplificação.

A amostra foi constituída por todas as idosas participantes do projeto NATI, nas modalidades de ginástica e hidroginástica com mais de 60 anos, totalizando 191 idosas. 
Foi entregue as participantes um Termo de Consentimento, que assegurava os objetivos do estudo, bem como a confidencialidade das identidades e a ausência de riscos na pesquisa.

Os dados coletados foram digitados em um banco e, para sua análise, foram utilizados procedimentos de estatística descritiva (valores de média e percentual).

\section{RESULTADOS E DISCUSSÃO}

Do total de 191 idosas participantes de atividades físicas nos projetos de ginástica, hidroginástica e musculação, foi observado que a grande maioria situa-se na faixa etária de 60 a 74 anos de idade, com nível socioeconômico equivalente as classes sociais C com 47,1\%, seguido de $24,6 \%$ nas classes D e E, demonstrado na (TAB. 1) a seguir.

\section{TABELA 1}

Características etárias (em anos) e socioeconômicas (ABEP 2009) das idosas participantes do Projeto NATI/ESEF/UFPel.

\begin{tabular}{|c|c|c|}
\hline Variável & N & \% \\
\hline Idade & & 23,6 \\
\hline $60-64$ & 45 & 27,7 \\
\hline $65-69$ & 53 & 23,6 \\
\hline $70-74$ & 45 & 17,3 \\
\hline $75-79$ & 33 & 7,8 \\
\hline 80 ou mais & 15 & $18,3 \%$ \\
\hline Classe Social & & $47,1 \%$ \\
\hline A e B & 35 & $24,6 \%$ \\
\hline C & 90 & $9,9 \%$ \\
\hline D e E & 47 & \\
\hline NI* & 19 & \\
\hline
\end{tabular}

*Não informado

Quanto às atividades de lazer preferidas pelas participantes do projeto, divididas por faixas etárias, as mesmas são especificadas na (TAB. 2) 
TABELA 2

Atividades de lazer preferidas pelas idosas participantes do NATI divididas por faixa etária da amostra.

\begin{tabular}{|c|c|c|c|c|c|c|}
\hline \multirow[t]{2}{*}{ ATIVIDADES* } & \multicolumn{5}{|c|}{ Faixa etária } & \multirow[b]{2}{*}{$\begin{array}{l}\text { Total } \\
\text { n }(\%)\end{array}$} \\
\hline & $\begin{array}{l}60-64 \\
\text { n (\%) }\end{array}$ & $\begin{array}{l}65-69 \\
\text { n }(\%)\end{array}$ & $\begin{array}{l}70-74 \\
\text { n }(\%)\end{array}$ & $\begin{array}{l}75-79 \\
\text { n }(\%)\end{array}$ & $\begin{array}{l}80-89 \\
\text { n }(\%)\end{array}$ & \\
\hline Assistir filmes & $8(17,7 \%)$ & $13(24,5 \%)$ & $6(13,3 \%)$ & $7(21,2 \%)$ & $3(20 \%)$ & $37(19,3 \%)$ \\
\hline Biscuit & $3(6,6 \%)$ & $3(5,6 \%)$ & $3(6,6 \%)$ & $1(3,03 \%)$ & $1(6,6 \%)$ & $11(5,7 \%)$ \\
\hline Bordados & $12(26,6 \%)$ & $12(22,6 \%)$ & $10(22,2 \%)$ & $9(27,2 \%)$ & $3(20 \%)$ & $46(24,08 \%)$ \\
\hline Costura & $11(24,4 \%)$ & $20(37,7 \%)$ & $12(26,6 \%)$ & $11(33,3 \%)$ & $5(33,3 \%)$ & $59(30,8 \%)$ \\
\hline Crochê & $10(22,2 \%)$ & $20(37,7 \%)$ & $13(28,8 \%)$ & $15(45,4 \%)$ & $6(40 \%)$ & $64(33,5 \%)$ \\
\hline Pintura em madeira & $15(33,3 \%)$ & $16(30,1 \%)$ & $10(22,2 \%)$ & $5(15,1 \%)$ & $2(33,3 \%)$ & $48(25,1 \%)$ \\
\hline Pintura em tecido & $14(31,1 \%)$ & $12(22,6 \%)$ & $10(22,2 \%)$ & $4(12,1 \%)$ & $4(26,6 \%)$ & $44(23,03 \%)$ \\
\hline Pintura em tela & $14(31,1 \%)$ & $9(16,9 \%)$ & $4(8,8 \%)$ & $2(6,06 \%)$ & $2(13,3 \%)$ & $31(16,2 \%)$ \\
\hline Tricô & $7(15,5 \%)$ & $4(7,5 \%)$ & $4(8,8 \%)$ & $2(6,06 \%)$ & $1(6,6, \%)$ & $18(9,4 \%)$ \\
\hline Outras Atividades $^{* *}$ & $9(20 \%)$ & $12(22,6 \%)$ & $9(20 \%)$ & $5(15,1 \%)$ & $4(26,6 \%)$ & $39(20,4 \%)$ \\
\hline Não opinaram & $5(11,1 \%)$ & $5(9,4 \%)$ & $9(20 \%)$ & $8(24,4 \%)$ & $2(13,3 \%)$ & $29(15,1 \%)$ \\
\hline
\end{tabular}

"Foi possível escolher mais de uma atividade " Ex.: baile, teatro, informática. Em alguns casos a idosa não exemplificou a atividade.

É notável a preferência das idosas por atividades manuais, especialmente, crochê, costura, pintura e bordados. Resultados semelhantes foram encontrados em outros estudos, como no caso da pesquisa realizada na cidade de Veranópolis - RS onde dos 121 idosos da amostra, 51,8\% das mulheres demonstrou interesse em atividades manuais como lazer (ARGIMON et al., 2004). Em outro estudo efetuado na cidade de Rio Claro - SP, dos 151 
idosos que compuseram a amostra, $45 \%$ relatou preferência por atividades manuais no seu lazer domiciliar (BALSAN, 2006).

Além das atividades manuais, nota-se uma grande preferência pelo lazer artístico, principalmente pintura em madeira e tecido. As atividades de pintura auxiliam os idosos no resgate de certa autonomia e auto-estima. Os idosos vivem a alegria de descobertas de potenciais que julgavam perdidos, e este resgate dinamiza a organização das percepções, sentimentos e sensações. Portanto, os conteúdos internos de sua vida psíquica são transformados em imagens e símbolos, facilitando o autorreconhecimento das potencialidades dos idosos, que ao se sentirem capazes de realizações pessoais, aumentam sua confiança (CAVALCANTI, et al. 2003; SCHAMBECK, 2004; PUFFAL; WOSIACK; BECKER JR, 2009).

Interessante observar que atividades esportivas ou que envolvam alguma atividade física foram pouco assinaladas pelas idosas. A despeito disso, é possível que as idosas realizem algum outro tipo de lazer em outros âmbitos, porém não consideram estas atividades como lazer. Por exemplo, as próprias atividades oferecidas pelo projeto NATI, as quais não foram consideradas como atividades de lazer pelas idosas. Rodrigues (2007) afirma que o lazer é um tema tão abrangente em significados na vida dos idosos que a maioria não possui uma resposta objetiva acerca do tema.

A lacuna existente no que diz respeito à significação do lazer para o idoso decorre de diversos fatores, dentre eles podemos citar: estereótipos, fator econômico, tempo disponível e o acesso ao espaço de lazer (RODRIGUES, 2002). Além dessas barreiras, outros determinantes dificultam na concretização do ideal de lazer pelo idoso, entre eles citamos a configuração do processo educativo, que não fornece o entendimento do lazer como fator 
importante no seu desenvolvimento humano e social, formando um adulto que não valoriza e não se interessa por este espaço (MORI; SILVA, 2010).

Outros fatores também afetam negativamente sobre a prática de lazer do idoso, como os preconceitos e estigmas sofridos pelos idosos devido a diminuição no nível de produção ligado ao trabalho, refletindo em uma menor aceitação de atividades de lazer por parte do idoso (MORI; SILVA, 2010). Nesta perspectiva, com o envelhecimento ocorre uma progressiva fragilização social do indivíduo, pressionando os sujeitos a serem mais modestos nas suas reivindicações de felicidade, contentando-se mais do que nunca a simplesmente evitar o sofrimento, ficando a tarefa de obter prazer em segundo plano. (MAFFIOLETTI, 2008).

Desta forma, para que o idoso se sinta motivado a realizar atividades em prol de si mesmo, torna-se imprescindível o apoio social, principalmente aquele advindo da família e dos amigos. Estas entidades são a base da rede social dos idosos e tendem a exercer grande influência sobre os comportamentos do idoso, principalmente pela facilidade de acesso ao mesmo, configurando-se como os principais fornecedores de apoio emocional e instrumental (BORRERO, 2008).

Ainda sobre nossos resultados, percebe-se que muitas das atividades citadas pelas idosas podem ser realizadas sem sair de seu domicílio. Este apontamento vai ao encontro dos resultados encontrados por Doimo, Dernt \& Lago (2008), que ao analisar o uso do tempo de um grupo de mulheres idosas, constatou que $74 \%$ das atividades diárias das idosas ocorriam dentro de seu próprio domicílio. Este mesmo estudo verificou, que o tempo livre é preenchido em grande parte na forma de lazer passivo, ou seja, atividades sedentárias ou pouco ativas físicamente. Resultados semelhantes também foram apreciados por Bacha e Strehlau (2010), que analisaram os hábitos de lazer de 1400 idosos de São Paulo, onde aproximadamente 16\% 
da amostra apresentou hábitos de lazer restritamente domiciliar, sendo que predominam em mulheres, viúvas, mais velhas, de renda intermediária (classes C e D) e escolaridade até ensino médio incompleto, características semelhantes na amostra aqui estudada.

Entretanto, essas características evidenciadas devem ser analisadas de forma cautelosa, pois encarar as dificuldades enfrentadas para o envolvimento do idoso em determinadas atividades como sendo apenas de responsabilidade dele próprio é errôneo. Estes aspectos são influenciados diretamente pelo ambiente que rodeia o idoso, de suas redes sociais e das próprias relações estabelecidas com as mesmas cotidianamente que, em alguns casos, tendem a isolar o idoso em ambientes cada vez mais separados do restante da população. Nesta conjuntura a área da educação física surge como uma importante promotora de bem estar e qualidade de vida, na medida que pode potencializar aspectos relacionados as mais diversas esferas do homem, desde aspectos biológicos como força e flexibilidade, até a inclusão do indivíduo em um ambiente onde ele pode se desenvolver conforme seus potenciais, sendo um sujeito ativo na construção de seus determinantes sociais (PICCOLO, 2011).

\section{CONSIDERAÇÕES FINAIS}

A partir da realização deste estudo, podemos perceber a importância das atividades de lazer para a terceira idade, bem como que a significação dada ao lazer é distinta para esta população. Com a chegada da terceira idade e juntamente com a aposentadoria, o tempo livre do idoso é maximizado, sendo assim o período que antes era ocupado por obrigações se transforma em tempo ocioso. Neste contexto as atividades de lazer vêm para preencher este tempo, remodelando os sentidos acerca do cotidiano da pessoa idosa, mantendo ou melhorando as capacidades cognitivas e físicas dos idosos, dependendo da intensidade e do tipo de atividade escolhida. 
Apesar dos diversos avanços tecnológicos percebidos nos últimos anos, ainda se faz necessário uma maior conscientização dos idosos sobre os benefícios que o lazer pode acarretar sobre os diversos domínios biopsicossociais. Portanto, um maior espaço para este tipo de atividades deve ser oportunizado para os idosos, a partir de políticas públicas que garantam melhores condições de sociabilidade para as pessoas idosas, através de uma maior atenção a saúde e qualidade de vida desta população. Espaços como as universidades devem cada vez mais abrir suas portas para atender e identificar as necessidades dos idosos, bem como enriquecer o conhecimento científico acerca dos efeitos do lazer na terceira idade.

\section{REFERÊNCIAS}

ABEP - Associação Brasileira de Empresas de Pesquisa - Avaliação Socioeconômica - 2009 - Disponível em: www.abep.org. Acesso em: 25 ago. 2010.

AFONSO, M. R. et al. Do diagnóstico à ação: Núcleo de Atividades para a Terceira Idade (NATI) - trajetória e construção. Revista Brasileira de Atividade Física \& Saúde. v. 15, n. 3, p. 180-182, 2010.

ARGIMON, I. I. L. et al. O impacto de atividades de lazer no desenvolvimento cognitivo de idosos. Revista Brasileira de Ciências do Envelhecimento Humano. v. 1, n. 1, p. 38-47, 2004.

BACHA, M. L.; STREHLAU V. I. Lazer na terceira idade: um estudo com diferentes classes socioeconômicas em São Paulo. Revista Brasileira de Ciências do Envelhecimento Humano, v. 7, n. 2, p. 217-228, 2010.

BALSAN, R. Atividades de lazer dos idosos na cidade: um estudo de caso na cidade de Rio Claro-SP. Geografando, v. 1, n. 2, p. 65-87, 2006.

BORRERO, C. L. C. Soporte social informal, salud y funcionalidad en el anciano. Hacia la promoción de la salud, v. 13, p. 42-58, 2008.

BRASIL, Decreto n. 2.528 de 19 de outubro de 2006. Ministério da Saúde, Brasília, 19 out. 2006.

BRASIL, Decreto n. 8.842 de 4 de janeiro de 1994. Ministério do Desenvolvimento Social e Combate à Fome, Brasília, 4 jan. 1994. 
BRASIL, Decreto n. 10.741 de $1^{\text {o }}$ de outubro de 2003. Senado Federal, Brasília, 1 out. 2004.

CAVALCANTI, A. M. T.; et al. Pode a arte ser terapêutica? Reflexões a partir do trabalho desenvolvido com pacientes da "terceira idade" no ateliê da vida do Instituto de Psiquiatria da UFRJ - IPUB. Revista de Terapia Ocupacional da Universidade de São Paulo, v. 14, n. 3, p. 118-22, 2003.

DAVIM, R. M. B.; et al. O lazer diário como fator de qualidade de vida: o que pensa um grupo da terceira idade. Ciência, Cuidado e Saúde, v. 2, n. 1, p. 19-24, 2003.

DOIMO, L. A.; DERNT, A. M.; LAGO O. C. O uso do tempo no cotidiano de mulheres idosas: um método indicador do estilo de vida de grupos populacionais. Ciência \& Saúde Coletiva, v. 13, n. 4, p. 1133-1142, 2008.

FENALTI, R. C. S.; SCHWARTZ, G. M. Universidade aberta à terceira idade e a perspectiva de ressignificação do lazer. Revista Paulista de Educação Física, v. 17, n. 2, p. 131-41, 2003.

GÁSPARI, J. C.; SCHWARTZ, G. M. O Idoso e a Ressignificação Emocional do Lazer. Psicologia: Teoria e Pesquisa, v. 21, n. 1, p. 69-76, 2005.

IBGE - Instituto Brasileira de Geografia e Estatística. Distribuição da população por sexo, segundo os grupos de idade. Disponível em: http://www.censo2010.ibge.gov.br/sinopse/webservice/frm_piramide.php?ano=2010\&codigo $=0 \&$ corhomem=88C2E6\& cormulher=F9C189\&wmaxbarra=180. Acesso em: 25 abr. 2012.

MAFFIOLETTI, V. L. R. Velhice e Família: Reflexões Clínicas. Psicologia ciência e Profissão, v. 25, n. 3, p. 336-351, 2008.

MARCELLINO, N. C. Estudos do lazer: uma introdução. 4. ed. Campinas: Autores associados, 2006. 114p.

MORI, G.; SILVA, L. F. Lazer na terceira idade: desenvolvimento humano e qualidade de vida. Revista Motriz, v. 16, n. 4, p. 950-957, 2010.

PICCOLO, G. M. Os caminhos dialéticos do envelhecimento e sua relação com a educação física contemporânea. Revista Brasileira de Geriatria e Gerontologia, v. 14, n. 1, p. 169177, 2011.

PINTO, L. M. S. M. Formação de educadores e educadoras para o lazer: saberes e competências. Revista Brasileira de Ciências do Esporte, v. 22, n. 3, p. 53-71, 2001.

PRADO, S. D.; SAYD, J. D. A pesquisa sobre envelhecimento humano no Brasil: grupos e linhas de pesquisa. Ciência \& Saúde Coletiva, v. 9, n. 1, p. 57-68, 2004.

PUFFAL, D. C.; WOSIACK, R. M. R.; BEKKER JR., B. Arteterapia: favorecendo a autopercepção na terceira idade. Revista Brasileira de Ciências do Envelhecimento Humano, v. 6, n. 1, p. 136-145, 2009. 
RODRIGUES, M. C. A configuração do lazer no espaço das universidades da terceira idade. 2007. 170 f. Tese (Doutorado) - Faculdade de Educação Física, Universidade Estadual de Campinas, Campinas, 2007.

. O lazer do idoso: barreiras a superar. Revista Brasileira de Ciência e Movimento, v. 10, n. 4, p. 105-108, 2002.

SCHAMBECK, L. D. Arte-terapia na terceira idade: busca da felicidade, prazer, integração e promoção da saúde. 2004. 82f. Monografia (Especialização) - Diretoria de Pós- Graduação, Universidade do Extremo Sul Catarinense, Criciúma, 2004.

SANTOS, P. L.; FORONI, P. M.; CHAVES M. C. F. Atividades físicas e de lazer e seu impacto sobre a cognição no envelhecimento. Medicina (Ribeirão Preto), v.42, n. 1, p. 54-60, 2009.

\section{Endereço dos Autores:}

Luiza Isnardi Cardoso Ricardo

Rua Luiz de Camões, 625, Bairro Cohab Tablada

CEP 96055-630 - Pelotas - RS

Endereço Eletrônico: luricaardo@gmail.com 